\section{Incidental Detection of Massive Left Ventricular Calcification by Myocardial Perfusion Imaging: A Case of Imaging Illustrations}

Robert T. Tung, M.D. ${ }^{1}$, Johannes Heyns, M.D. ${ }^{2}$

Department of Veterans Affairs, Eastern Kansas HealthCare System, Topeka, KS

${ }^{1}$ Cardiology Section

${ }^{2}$ Radiology Department

Received April 10, 2021; Accepted for publication May 25, 2021; Published online Oct. 14, 2021 htlps: doi.org 10.17161 lijm.voll4.15359

\section{INTRODUCTION}

Cardiac calcification is not uncommon, but massive left ventricular (LV) myocardial calcification is rare and can be an uncommon cause of congestive heart failure., ${ }^{1,2}$ Myocardial calcification can be associated with metastatic deposition, infarction, or other endocrine disorders. ${ }^{3}$ It represents abnormal accumulation of calcium salts in the myocardium and includes two basic forms: dystrophic and metastatic. ${ }^{4}$ Dystrophic calcification is more prevalent than metastatic calcification. It represents deposition of calcium salts in previously damaged tissue with normal calcium metabolism and is seen commonly in patients with myocardial infarction or primary myocardial disease. ${ }^{5}$

Metastatic calcification is caused by deposition of calcium salts in previously normal tissue due to disturbance in calcium or phosphorus metabolism seen in disorders like hyperparathyroidism, chronic renal disease, widespread bone destruction from metastases, or myeloma. ${ }^{3}$ Depending on the degree of calcification, it can be detected by chest x-ray, echocardiography, or computed tomography (CT) of the chest. Chest $\mathrm{CT}$ is the optimal modality for identifying and characterizing myocardial calcifications as illustrated in this case. Myocardial calcification is an important marker of underlying pathology and knowledge of its potential etiology are important to provide accurate differential diagnosis.

We report a case with illustrated images of massive LV calcification detected incidentally during regadenoson single photon emission computed tomography (SPECT) myocardial perfusion imaging (MPI) and confirmed by chest computed tomography.

\section{CASE REPORT}

This case was a 70-year-old patient who had a history of atrial fibrillation (AF), symptomatic of dyspnea and congestive heart failure. At that time, his AF was converted by electrical cardioversion with resolution of his symptoms. A year later, he presented with several months' history of progressive and marked dyspnea with minimal exertion. He underwent regadenoson MPI for cardiac evaluation. His electrocardiogram (ECG) showed a normal sinus rhythm, first degree atrioventricular (AV) block, and T wave inversion in the inferolateral leads suggestive of myocardial ischemia (Figure 1). His MPI demonstrated mildly decreased LV ejection fraction of $49 \%$ without evidence of myocardial ischemia or infarction; however, significant LV

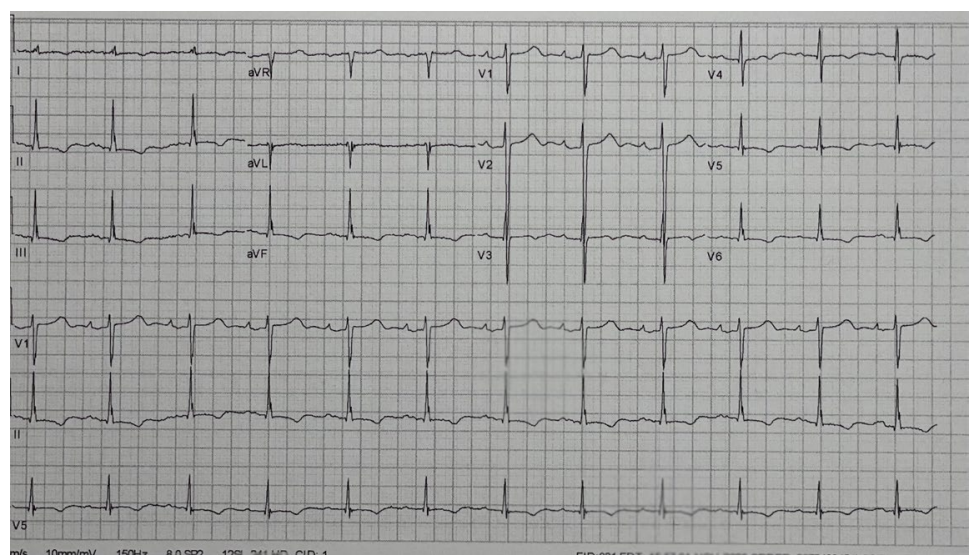

Figure l. The electrocardiogram showed normal sinus rhythm, first-degree AV block, and T-wave inversion in the inferolateral leads.

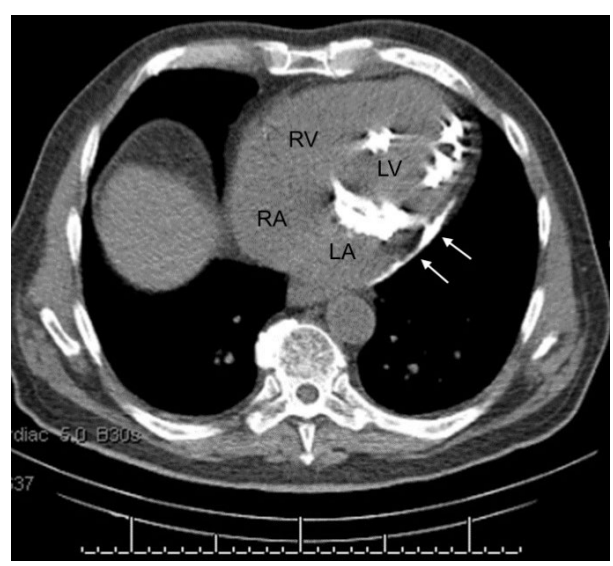

Figure 2. The axial image of low-dose CT during MPI demonstrated a significant amount of calcification at mitral valvular annulus, LV myocardium at septum, and free wall as well as pericardium (white arrows). [LA = left atrium; $\mathrm{LV}=$ left ventricle; $\mathrm{RA}$ = right atrium; $\mathrm{LV}=$ left ventricle.]

calcification (Figure 2) was observed on the low-dose CT used for attenuation correction during MPI.

Subsequently, other imaging studies, including chest x-ray, echocardiography, and chest CT, were obtained for further evaluation. The chest x-ray (Figure 3) showed extensive cardiac calcification, and the echocardiogram (Figure 4) revealed significant calcification of mitral valve annulus, LV septum, and mid free wall. However, his chest CT (Figure 5) revealed massive myocardial calcification involving only LV and its overlaying pericardium.

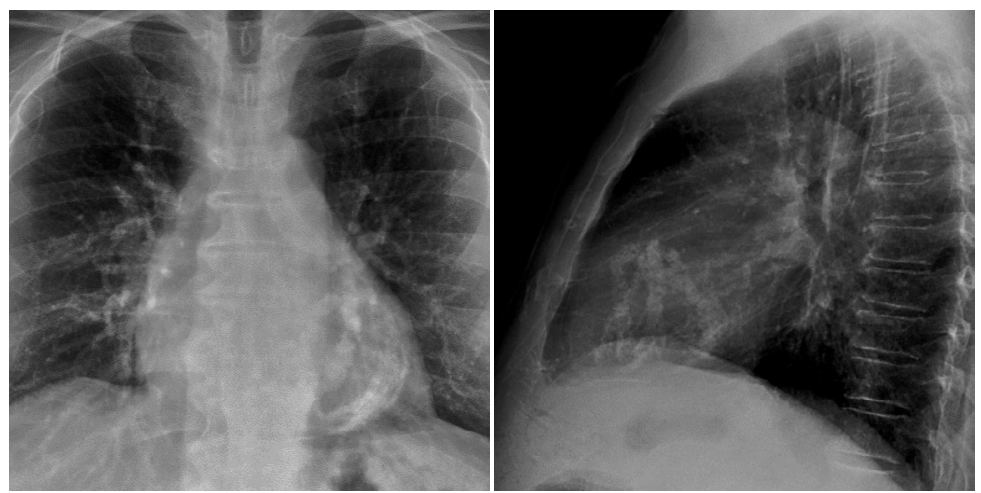

Figure 3. The posteroanterior (left) and lateral (right) views of patient's chest x-ray. 


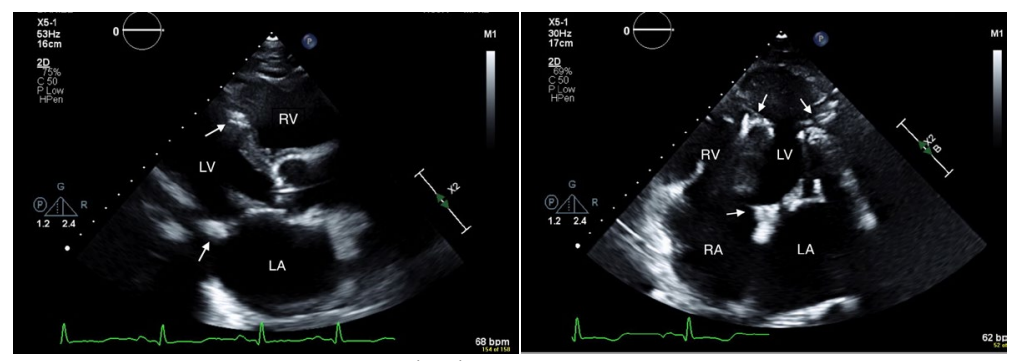

Figure 4. The parasternal view (left) and 4-chamber view of echocardiogram (right) showed calcification of mitral annulus, LV septum, and free wall (indicated by white arrows). [LA = left atrium; $\mathrm{LV}=$ left ventricle; $\mathrm{RA}=$ right atrium; $\mathrm{LV}=$ left ventricle.]

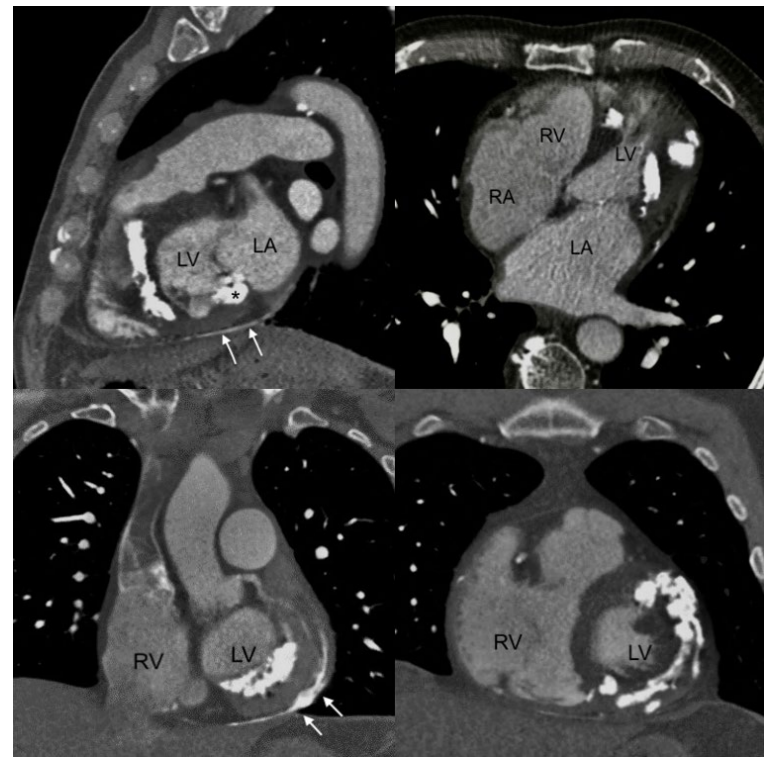

Figure 5. The sagittal (upper left), axial (upper right), and coronal images (lower left and right) of the chest CT demonstrated calcification of LV myocardium, mitral valve annulus (* in upper left image), and pericardium (white arrows in upper and lower left images) over the LV. [LA = left atrium; $L V=$ left ventricle; $\mathrm{RA}=$ right atrium; $\mathrm{LV}=$ left ventricle.]

The patient had no past history of malignancy, endocrine disorder, tuberculosis, or recent traveling to other countries. Extensive laboratory evaluation revealed elevated NT-proB-type Natriuertic Peptide (1113.5 pg/ml; normal < $100 \mathrm{pg} / \mathrm{ml}$ ) and C-reactive protein (1.21 mg/ $\mathrm{dL}$, normal $<0.5 \mathrm{mg} / \mathrm{dL}$ ), but normal findings of complete blood count, basic chemistry profile, troponin-I, sedimentation rate, antinuclear antibody, antimitochondrial antibody, serum calcium/phosphate level, vitamin D level, and thyroid and parathyroid hormone levels. His echocardiography showed: (1) normal LV systolic function with calcification in the LV septum, mid free wall and mitral valve annulus (Figure 4) and normal right ventricular (RV) size and systolic function; (2) moderate bi-atrial enlargement with mild mitral regurgitation, moderate tricuspid regurgitation; and (3) severe pulmonary hypertension and LV diastolic dysfunction. Cardiac catheterization demonstrated: (1) no angiographic evidence of coronary artery disease; (2) moderately severe pulmonary hypertension with pulmonary artery systolic pressure of $67 \mathrm{mmHg}$; (3) elevated pulmonary capillary wedge pressure, measured at $28 \mathrm{mmHg}$; (4) elevated LV end-diastolic pressure, measured $29 \mathrm{mmHg}$; and (5) no evidence of ventricular interdependence on simultaneous $\mathrm{LV} / \mathrm{RV}$ pressure measurement.

The final diagnosis was congestive diastolic heart failure due to massive LV myocardial calcification of unknown etiology. He was treated with aggressive diuresis and other guideline-driven medical
KANSAS JOURNAL of MED I C INE

LEFT VENTRICULAR CALCIFICATION

continued.

therapy successfully with marked improvement of his dyspnea on exertion during follow-up.

\section{DISCUSSION}

The SPECT MPI is a well-established, commonly used diagnostic test for evaluation of coronary artery disease (CAD). ${ }^{6}$ To improve quality of the study, low-resolution CT is used commonly for artifact attenuation and anatomical correction during SPECT MPI. ${ }^{7}$ The low-resolution, unenhanced CT portion of the MPI is considered low-quality, therefore, labelled by manufacturers as "non-diagnostic". In many centers, it is used only for image fusion and attenuation correction, and these images are not reviewed routinely or reported by cardiologists. Incidental findings on low-resolution CT during cardiac MPI are frequent, but clinically significant ones are relatively infrequent $^{8}$; however, some might be of important clinical significance. ${ }^{8-11}$

There is no uniform or consensus recommendations of reporting incidental findings during cardiac CT imaging. They ranged from no recommendations, ${ }^{12,13}$ to optional, ${ }^{14}$ to recommended or mandatory reporting. ${ }^{15,16}$ This report described, for the first time, the incidental detection of a massive LV myocardial calcification by low-resolution CT during cardiac MPI in a patient presented as congestive heart failure. Therefore, it was appropriate and necessary to review low-resolution CT images during cardiac MPI to detect incidental but clinically significant findings.

\section{CONCLUSIONS}

Massive LV myocardial calcification is rare and can be an uncommon cause of congestive heart failure. To ensure high-quality patient care, low-resolution CT used for attenuation correction during cardiac MPI should be reviewed routinely and interpreted to identify incidental but clinically significant findings as demonstrated by this case.

\section{REFERENCES}

${ }^{1}$ Torfs M, Salgado R, Van Herck P, Corthouts B, Robert D, Parizel PM. A curious case of acute myocardial calcifications. Circulation 2016; 133(10):e426-427. PMID:26951826.

El-Bialy A, Shenoda M, Saleh J, Tilkian A. Myocardial calcification as a rare cause of congestive heart failure: A case report. J Cardiovasc Pharmacol Ther 2005; 10(2):137-143. PMID: 15965565.

Nance JW, Crane GM, Halushka MK, Fishman EK, Zimmerman SL. Myocardial calcifications: Pathophysiology, etiologies, differential diagnoses, and imaging findings. J Cardiovasc Comput Tomogr 2015; 9(1);58-67. PMID: 25456525 .

${ }^{4}$ Perkins JA. Tissue renewal, regeneration, and repair. In: V Kumar, AK Abbas, N Fausto, JC Aster (Eds.). Robbins and Cotran Pathologic Basis of Disease. Eighth Edition. Philadelphia: WEB Saunders, 2010, pp 79-110. ISBN: 9781455753406.

Shackley BS, Nguyen TP, Shivkumar K, Finn PJ, Fishbein MC. Idiopathic massive myocardial calcification: A case report and review of the literature. Cardiovasc Pathol 2011; 20(2):e79-83. PMID:20598909.

${ }^{6}$ Hendel RC, Berman DS, Di Carli MF, et al. ACCF/ASNC/ACR/AHA/ ASE/SCCT/SCMR/SNM 2009 appropriate use criteria for cardiac radionuclide imaging: A report of the American College of Cardiology Foundation Appropriate Use Criteria Task Force, the American Society of Nuclear Cardiology, the American College of Radiology, the American Heart Association, the American Society of Echocardiography, the Society of Cardiovascular Computed Tomography, the Society for Cardiovascular Magnetic Resonance, and the Society of Nuclear Medicine. Circulation 2009; 119(22):e561-587. PMID: 19451357. 


\section{KANSAS JOURNAL of MEDICINE}

LEFT VENTRICULAR CALCIFICATION

continued.

7 Hendel RC, Corbett JR, Cullom J, DePuey EG, Garcia EV, Bateman TM. The value and practice of attenuation correction for myocardial perfusion SPECT Imaging: A joint position statement from the American Society of Nuclear Cardiology and the Society of Nuclear Medicine. J Nucl Cardiol 2002; 9(1):135-143. PMID: 11845140.

${ }^{8}$ Coward J, Nightingale J, Hogg P. The clinical dilemma of incidental findings on the low-resolution CT images from SPECT/CT MPI studies. J Nucl Med Technol 2016; 44(3):167-172. PMID: 27102662.

9 Osman MM, Cohade C, Fishman E, Wahl RL. Clinically significant incidental findings on the unenhanced CT portion of PET/CT studies: Frequency in 250 patients. J Nucl Med 2005; 46(8):1352-1355. PMID: 16085594.

${ }^{10}$ Goetze S, Pannu HK, Wahl RL. Clinically significant abnormal findings on the "non-diagnostic" CT portion of low-amperage-CT attenuationcorrected myocardial perfusion SPECT/CT studies. J Nucl Med 2006; 47(8):1312-1318. PMID: 16883011.

11 Tung RT, Heyns J, Dryer L. Incidental findings of pulmonary and hilar malignancy by low-resolution computed tomography used in myocardial perfusion imaging. Fed Pract 2020; 37(Suppl 2):S27-21. PMID: 32952384.

${ }_{12}$ Holly TA, Abbott BG, Al-Mallah M, et al. Single photon-emission computed tomography. J Nucl Cardiol 2010; 17(5):941-973. PMID: 20552312.

13 Dorbala S, Ananthasubramaniam K, Armstrong IS, et al. Single photon emission computed tomography (SPECT) myocardial perfusion imaging guidelines: Instrumentation, acquisition, processing, and interpretation. J Nucl Cardiol 2018; 25(5):1784-1846. PMID: 29802599.

14 Tilkermeier P, Bourque J, Doukky R, Sanghani R, Weinberg R. ASNC imaging guidelines for nuclear cardiology procedures: Standardized reporting of nuclear cardiology procedures. J Nucl Cardiol 2017; 24(6):2064-2128. PMID: 28916938.

${ }^{15}$ Dorbala S, DiCarli MF, Delbeke D, et al. SNMMI/ASNC/SCCT guideline for cardiac SPECT/CT and PET/CT 1.O. J Nucl Med 2013; 54(8):14851507. PMID: 23781013.

${ }^{16}$ Dilsizian V, Bacharach SL, Beanlands RS, et al. ASNC imaging guidelines/SNMMI procedure standard for positron emission tomography (PET) nuclear cardiology procedures. J Nucl Cardiol 2016; 23(5):1187-1226. PMID: 27392702.

Keywords: vascular calcification, myocardial perfusion imaging, SPECT CT, incidental findings 